\title{
Automatic Computation of Average Brain Models
}

\author{
Alexandre Guimond ${ }^{1,2}$, Jean Meunier ${ }^{2}$, and Jean-Philippe Thirion ${ }^{1 \star}$ \\ 1 INRIA Sophia Antipolis, Epidaure Project 2004, France \\ \{guimond, thirion\}osophia.inria.fr \\ http://www.inria.fr/Equipes/EPIDAURE-eng . html \\ 2 Université de Montréal, Département d'informatique et de recherche opérationnelle, \\ Montréal, Canada. \\ meunier@iro.umontreal.ca \\ http://www. IRO. UMontreal.CA/labs/vision
}

\begin{abstract}
We present a completely automatic method to build average anatomical models of the human brain using a set of MR images. The models computed present two important characteristics: an average intensity and an average shape. We provide results showing convergence toward the barycenter of the image set used for the computation of the model.
\end{abstract}

\section{Introduction}

Someone suffering from a neurological disorder such as epilepsy or schizophrenia will usually undergo a series of tests to assess the anatomy and the functional activity of his or her brain. The results of these tests are then analyzed to identify if abnormal variations are present, providing valuable information for future medical treatment.

An important tool used to diagnose abnormal anatomical variations are medical atlases. Traditional ones $[14,12]$ are presented in textbooks, but computerized atlases comprising information in a more practical and quantitative manner are becoming available [10]. They also usually include information obtained from a set of subjects [8] instead of a single individual, making them more representative of a population and enabling the calculation of normal variations [17].

The following work aims to develop and validate the concepts introduced in a previous paper [9] to build an average model of the human brain using a set of magnetic resonance (MR) images obtained from normal subjects. We intend to fabricate an image with two important characteristics: average tissue intensity and average tissue shape up to an affine transformation.

As depicted in Fig. 1, our method can be summarized in the following manner. Affine registration between all the images of the set and a reference image corrects for differences due to translations, rotations, scalings and shearings. These are morphometrical variations that are not of concern for our study. Elastic registration is then used to evaluate residual variations due to pure morphological

\footnotetext{
* Now in Focus Imaging, Sophia Antipolis, France
} 


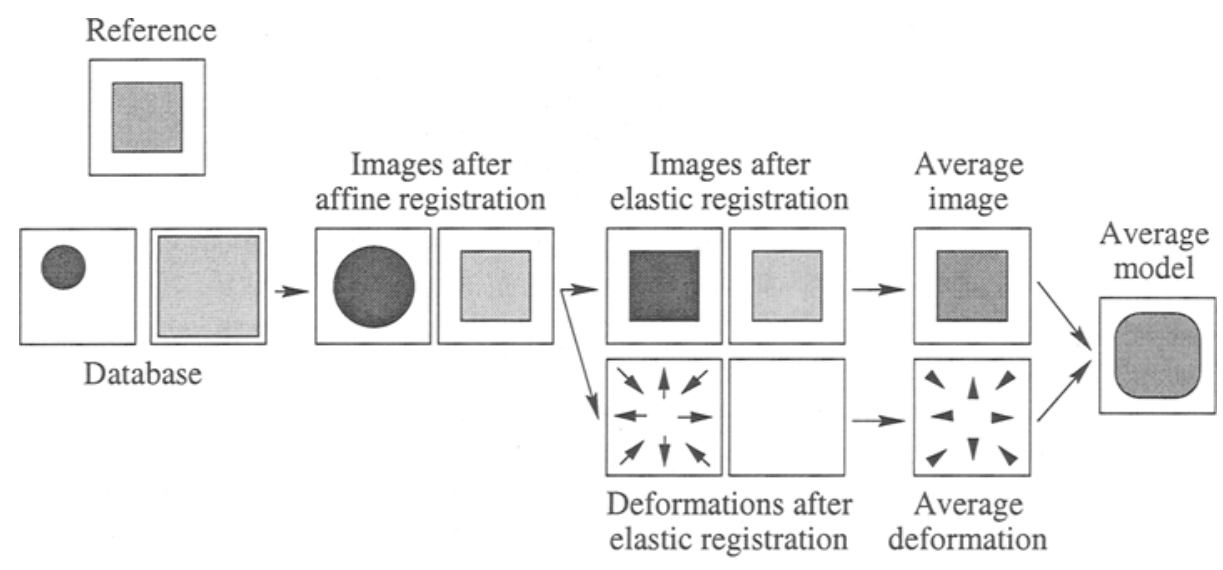

Fig. 1. Average model construction method.

differences and produce images having the same shape as the reference. The images and the residual deformations are averaged and the average deformation applied to the average image to produce the model. It presents an average intensity and an average shape modulo an affine transformation corresponding to the affine characteristics of the reference image.

The main contribution of this paper is the description of a fully automatic technique to obtain an average intensity image combined with an average shape image, producing the average model $M$.

The most similar work regarding average intensity atlases is that of [3] who created from nine MR scans a two-dimensional image representing the average intensity of the mid-sagittal plane. Thirteen manually identified landmarks in the mid-sagittal plane of each scan where matched with a reference image using the thin-plate spline interpolant [2]. The nine resampled images where then averaged to result into a morphometric average atlas. Our method differs mainly by two aspects. First, as suggested by [3], we make full use of the three-dimensionality of the scans to compute a three-dimensional average image. Second, our registration method is automatic and computes a dense deformation field instead of an interpolated function based on thirteen landmarks. This deformation identifies for each voxel of the reference the corresponding positions in the other scans. Within this process, every voxel of the reference can be though of as a landmark automatically determined in the other scans.

The work of [8], where three hundred and five (305) three-dimensional MR scans were registered using translations, rotations and scalings, and averaged to build a statistical neuroanatomical model, also relates to our work. We follow the same idea but proceed further by using a less constrained type of deformation.

As will be shown, compared to these previous efforts, our method provides clearer images with higher contrasts and more sharp definitions of tissue boundaries.

The average shape concept is most similar to the work of [13], [4] and [11] who compute average shapes modulo similarity or affine transformations. We have not 
tried to strictly follow the theory developed in their works. Our intention was to conform to the idea of making abstraction of differences between images due to first order transformations, and analyze residual variations. Our main contribution resides in the characteristics used to build the average shape, that is the image intensities instead of landmarks or crestlines. Again, this enables the computation of dense deformations fields representing variations everywhere is the MR scan, as opposed to interpolating transformations found using landmarks, lines or surfaces. We believe this technique may find less accurate matches in the close surroundings of the landmarks, but provides better overall registration.

The remaining sections of this paper are organized in the following manner. First, we detail the method used to construct the average model. We then present results showing the convergence of the method towards an average intensity and an average shape, and show the effect of the choice of reference image. We conclude by a discussion on future research tracks.

\section{Methodology}

\subsection{Registration}

The work that follows assumes each point in one image has a corresponding equivalent in the others. It also assumes available a matching method able to find these correspondences and capable of providing a vector field representing those relationships. In theory, neither of these conditions is realized. That is, at a microscopic scale, there is not a one to one relationship between the brain cells of two individuals, and assuming there was, to this day, no algorithm is able to find it. In practice however, deforming one brain so its shape matches the one of another is conceivable and many algorithms realizing this process have been developed $[2,1,6,7,16]$.

The procedure used in the following work is the demons method [15] which is a fully automated intensity-based registration method. It provides results qualitatively similar to [1] and [6] but with an implementation one or two orders of magnitude faster. From a practical point of view, it is worth mentioning that although the algorithm matches intensities and that a global intensity correction is made over the whole image, the transformed image of $I_{1}$ is not an exact duplicate of $I_{2}$. This is due to the smoothness constraint applied to the displacement field which establishes a compromise between intensity resemblance and uniform local deformations.

\subsection{Average Model Construction}

The average model construction (See Fig. 2) needs as input a reference image $I_{R}$ and a set of $N$ images $I_{1}, \ldots, I_{N}$ representing the group of subjects under consideration. The method can be divided in six steps as follows:

1. The first step regards the evaluation of shape differences between the reference and each image of the set. Elastic registration between $I_{R}$ and $I_{i}$ provides vector fields $D_{i}$ giving for each voxel $x_{R}$ of $I_{R}$ the analogous anatomical location $x_{i}$ in $I_{i}$. 


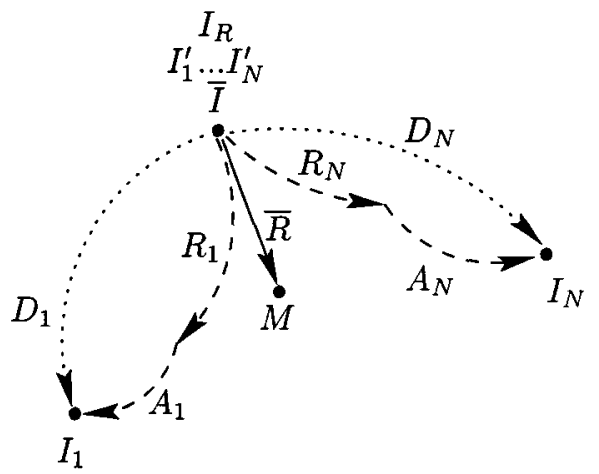

Fig. 2. Average model construction. First step (dotted arrows): the $D_{i}$ are obtained. Second step: $I_{i}$ are resampled into $I_{i}^{\prime}$. Third step: $\bar{I}$ is computed. Fourth step (dashed arrows): $D_{i}$ are decomposed in $A_{i}$ and $R_{i}$. Fifth step: $\bar{R}$ is computed. Sixth step (full line): Combination of average intensity and shape to obtain the average model $M$.

2. These correspondences found, the second step resamples the $I_{i}$ using trilinear interpolation to provide $I_{i}^{\prime}$ presenting the same shape as $I_{R}$ with the intensities of $I_{i}$.

3. In the third step, the $I_{i}^{\prime}$ are averaged pixelwise, producing a mean intensity image $\bar{I}$ with the shape of $I_{R}$.

4. The fourth step concerns the decomposition of $D_{i}$ into affine $\left(A_{i}\right)$ and residual $\left(R_{i}\right)$ components. Since we want correspondences between anatomical points of the $I_{i}$ and $I_{R}$ that have the form $x_{i}=D_{i}\left(x_{R}\right)=A_{i}\left(R_{i}\left(x_{R}\right)\right)$, we compute the $A_{i}$ by minimizing the distance $\sum_{x}\left\|x-A_{i}^{-1}\left(D_{i}(x)\right)\right\|^{2}$, where the summation is performed on the voxels positions in $I_{R}$ corresponding to cerebral tissues ${ }^{1}$. The residual components are obtained by computing $R_{i}(x)=A_{i}^{-1}(D(x))$.

5. The fifth step aims to produce the deformation presenting the shape variations between $I_{R}$ and the average shape of the set elements after correction of affine differences. Since the residual deformations $R_{i}$ are all defined in the same anatomical space, that of $I_{R}$, calculating their vectorwise average $\bar{R}(x)=1 / N \sum_{i}^{N} R_{i}(x)$ will provide the desired deformation.

6. The sixth and final step consists of applying this average residual deformation to the average intensity image to obtain an average intensity and average shape image representing the anatomical average model $M$.

Considering numerical errors due the fact that automatic registration methods usually perform better when images are closer to each other, all these steps may be repeated by replacing $I_{R}$ with $M$, thus constructing a model with a reference image closer to the barycenter of our set. Intuitively, this should reduce the mean registration error and provide a new model $M^{\prime}$ closer to the theoretical solution.

1 These positions are obtained using an automatic method for brain segmentation similar to that of [5]. From hereon, all summations over $x$ are assumed to be on the voxel positions obtained using this algorithm. 


\section{Results}

The method is tested by computing four models using two reference images $I_{R_{1}}$ and $I_{R_{2}}$ (See Figs. 5(a) and 5(b)) and two image sets $S_{1}$ and $S_{2}$, each composed of five images (See Table 1).

\begin{tabular}{|c|c|c|}
\hline Model & Reference & Image Set \\
\hline $\mathbf{M}_{11}$ & $I_{R_{1}}$ & $S_{1}$ \\
\hline $\mathbf{M}_{21}$ & $I_{R_{2}}$ & $S_{1}$ \\
\hline $\mathbf{M}_{12}$ & $I_{R_{1}}$ & $S_{2}$ \\
\hline $\mathbf{M}_{22}$ & $I_{R_{2}}$ & $S_{2}$ \\
\hline
\end{tabular}

Table 1. References and image sets used to build the different models.

The 3D MR protocol provides coronal images obtained using a 1.5 Tesla SIGNA (General Electric, Milwaukee, U.S.A.) whole body MR imaging system. One hundred and twenty four (124) coronal T1-weighted images were obtained using a spoiled gradient echo (SPGR) pulse sequence (TE $=9$ seconds, $\mathrm{TR}=34$ seconds, flip angle $=45^{\circ}$ ). Two NEX acquisitions took $27 \mathrm{~min}-$ utes and 52 seconds. The Field of View (FOV) of the images was $20 \mathrm{~cm}$ and each image refers to a contiguous section of tissue of $1.6 \mathrm{~mm}$ thickness. The $256 \times 256 \times 124$ voxels of size $0.78 \mathrm{~mm} \times 0.78 \mathrm{~mm} \times 1.6 \mathrm{~mm}$ were trilinearly interpolated to $200 \times 200 \times 198$ to give cubic voxels of $1 \mathrm{~mm}$ side.

We analyze our results with regards to two factors. First, the iteration process is investigated to see if convergence is achieved, and if so how fast is the convergence rate. Second, we study the effect of changing the reference image. If the model is a veritable average of the image set, changing the reference should produce an identical model up to an affine transformation defined by the affine difference between references.

In our evaluation procedure, three metrics are used. The first determines the average distance from an image $I$ to the elements of a set $S_{j}, \mathrm{AD}\left(I, S_{j}\right)=$ $\sqrt{\frac{1}{n} \sum_{x} \frac{1}{N} \sum_{i=1}^{N}\left\|x-R_{i}(x)\right\|^{2}}$, where $R_{i}$ is the residual deformation from $I$ to the $i$ th element of $S_{j}, n$ is the number of voxels characterizing cerebral tissues and $N$ represents the number of elements in $S_{j}$. The second is the root mean square (RMS) norm which supplies information regarding the shape variation expressed by a deformation field $D, \operatorname{RMSn}(D)=\sqrt{\frac{1}{n} \sum_{x}\|x-D(x)\|^{2}}$. The third provides a measure of brightness disparity between two images $I_{i}$ and $I_{j}$. It is the RMS difference of the images intensities at corresponding locations, $\operatorname{RMSd}\left(I_{i}, I_{j}\right)=\sqrt{\frac{1}{n} \sum_{x}\left(I_{i}(x)-I_{j}(x)\right)^{2}}$.

\subsection{Effect of Iterating}

To evaluate the effect of iterating, we construct the four models repeating the process five times and using the result of the previous iteration as the reference image. We will designate the model $M_{j k}$ computed at the $i$ th iteration by $M_{j k}^{(i)}$. For convenience, $M_{j k}^{(0)}$ will be identified to the average intensity image having the shape of $I_{j}$. 
Four measures were computed:

$\operatorname{AD}\left(M_{j k}^{(i)}, S_{k}\right)$ The average distance from the reference of the current iteration to all the elements of the set.

$\operatorname{RMSn}\left(\bar{R}_{j k}^{(i)}\right)$ The shape variation expressed by the residual deformation field $\bar{R}_{j k}^{(i)}$ when $M_{j k}^{(i)}$ is used as the reference.

$\operatorname{RMSn}\left(D_{j k}^{(i)}\right)$ The shape difference between models computed at successive iterations. $D_{j k}^{(i)}$ is the deformation obtained by registering $M_{j k}^{(i)}$ with $M_{j k}^{(i+1)}$. $\operatorname{RMSd}\left(M_{j k}^{(i)}, M_{j k}^{(i+1)}\right)$ The brightness disparity between models obtained at successive iterations.

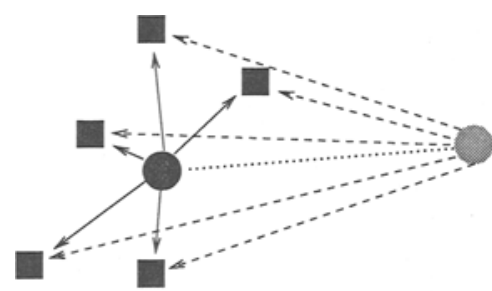

(a) The average distance of the model to the image set elements decreases.

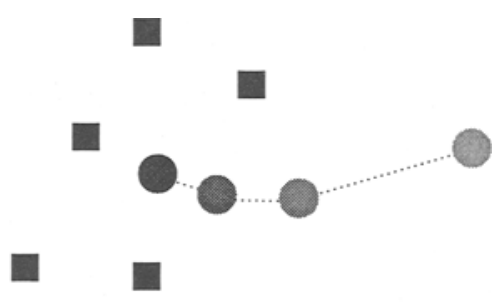

(b) The distance between successive models diminishes.

Fig. 3. Evolution of the model (circles) toward the center of the image set (squares).

If the models computed tend towards the barycenter of the image set, the first measure should diminish. This process is depicted in Fig. 3(a): as the model evolves towards the center (dotted line), the average distance to the image set elements decreases. The second and third measures, representing the shape evolution of the model (See Fig. 3(b)), should tend towards zero. Finally, the fourth value should also decrease to zero since it represents the brightness differences between successive models.

The results of these calculations on the four models are presented in Fig. 4. Note that the iterations range up to 4 and not 5 since we compare models computed at iterations $i$ and $i+1$. We remind the reader that "models" $M_{j k}^{(0)}$, that is models before the first iteration, characterize only average intensities and not average shapes.

From Fig. 4(a), we know the average distance from the references to the image set elements is close to $4.0 \mathrm{~mm}$ and reduces to about $2.5 \mathrm{~mm}$ when the model gets to the center of the image set, that is when the average shape is obtained. Compared to these values, the variation between successive models (See Figs. 4(b) and 4(c)), which is about $0.5 \mathrm{~mm}$, seems minor. Figure 4(d) presents numbers showing the brightness difference between successive models diminishes rapidly, increasing our belief that models do not evolve significantly after the first iteration. 


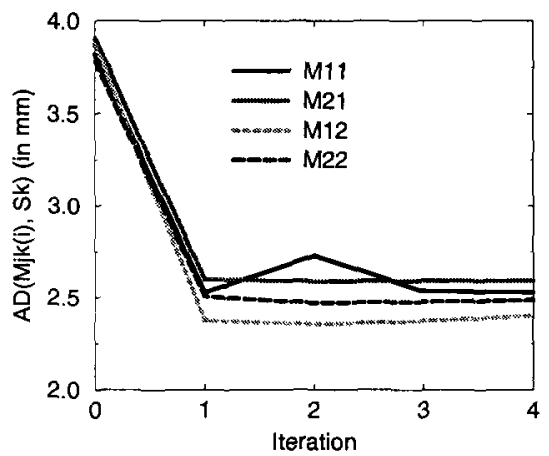

(a) Average distance to the reference of the current iteration.

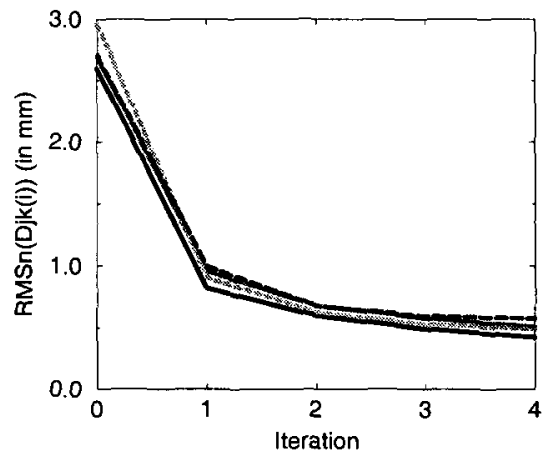

(c) Shape difference between models computed at successive iterations.

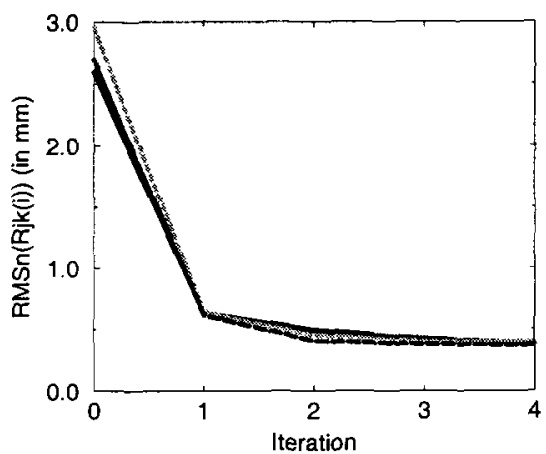

(b) Shape variation of the reference for the current iteration.

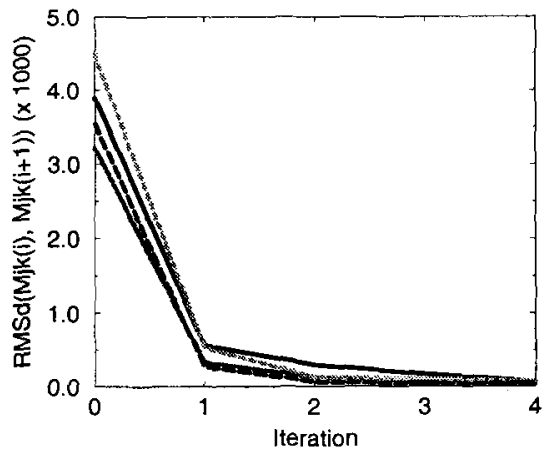

(d) Brightness disparity between models computed at successive iterations. Values are multiplied by 1000 .

Fig. 4. Impact of the iteration process when computing the models. Note that the iterations range up to 4 and not 5 since we compare models computed at iterations $i$ and $i+1$. We remind the reader that "models" $M_{j k}^{(0)}$, that is models before the first iteration, characterize only average intensities and not average shapes. 


\subsection{Effect of the Reference}

If the models computed are equal up to an affine transformation, changing the reference image should produce a model identical to the previous one after removing their affine differences. To verify this characteristic, we performed an affine registration between models built using the same image set. $M_{21}^{(i)}$ is registered with $M_{11}^{(i)}$ to provide the image $M_{21}^{\prime(i)}$ (See Fig. 5(d)) and $M_{22}^{(i)}$ with $M_{12}^{(i)}$ to result in $M_{22}^{\prime(i)}$.

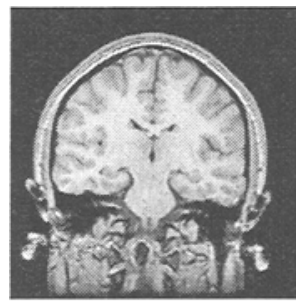

(a) A coronal slice of $I_{R_{1}}$.

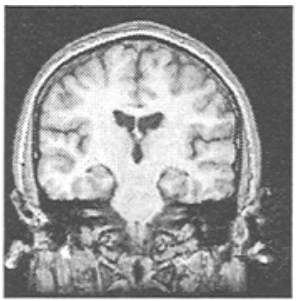

(b) A coronal slice of $I_{R_{2}}$.

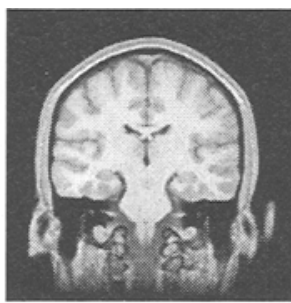

(c) A coronal slice of $M_{11}^{(5)}$.

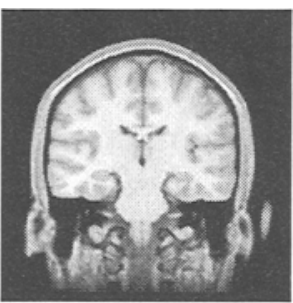

(d) A coronal slice of $M_{21}^{\prime(5)}$

Fig. 5. Reference images and models computed using the same image set. Figures 5(a) and 5 (b) present coronal slices from the two reference images $I_{R_{1}}$ and $I_{R_{2}}$ respectively. Figures 5(c) and 5(d) are slices of the models computed using these references. These two slices are taken at the same anatomical location in each model and can therefore be compared directly. The reader should observe the ventricular shape bias introduced using $I_{R_{2}}$ is minimal if not null. If familiar with the work of Bookstein (1991) and Evans et al. (1993), he or she will also appreciate the high contrast and visual quality of the images produced.

Two measure were used:

$\operatorname{RMSn}\left(D_{k}^{(i)}\right)$ The shape variation from $M_{2 k}^{(i)}$ to $M_{1 k}^{\prime(i)} . D_{k}^{(i)}$ is the deformation obtained by registering the two images.

$\operatorname{RMSd}\left(M_{1 k}^{(i)}, M_{2 k}^{(i)}\right)$ The brightness disparity between the two models.

Results are show in Figs. 6(a) and 6(b) respectively. We notice that shape variation between the models reduces from $3.0 \mathrm{~mm}$ to $1.0 \mathrm{~mm}$. This last value is close to the difference between successive models which we know from Figs. 4(b) and $4(\mathrm{c})$ to be approximately $0.5 \mathrm{~mm}$. The brightness disparity also diminishes rapidly and does not change drastically after the first iterations.

\section{Discussion}

Figure 4 presents numbers showing that our method constructs average models well representing the average intensity and shape of our image sets. In particular, Fig. 4(a) shows that the average distance from one image to the set elements is about $4.0 \mathrm{~mm}$. This distance reduces and stays at approximately $2.5 \mathrm{~mm}$ after the first iteration. Figures 4(b) and 4(c) illustrate a minor shape evolution of 


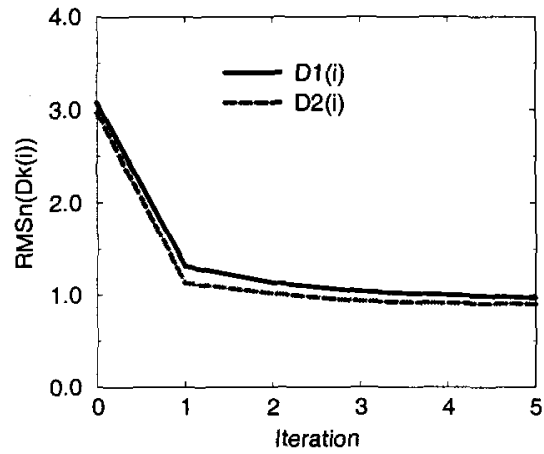

(a) Shape variation between $M_{1 k}^{(i)}$ and $M_{2 k}^{\prime(i)}$.

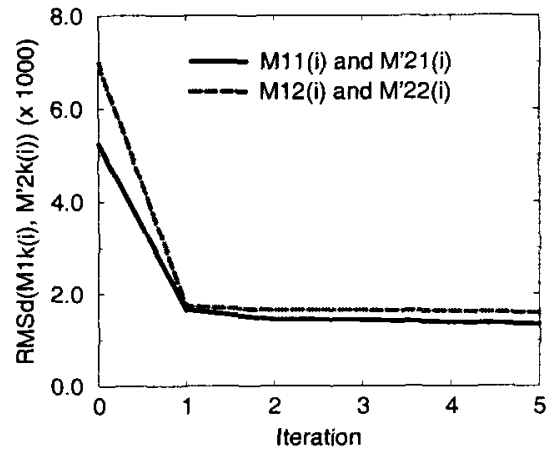

(b) Brightness disparity between $M_{1 k}^{(i)}$ and $M_{2 k}^{\prime(i)}$. Values are multiplied by 1000 .

Fig. 6. Influence of the reference on the model computed.

the models at each iteration, we believe due to numerical errors. Furthermore, Fig. 4(d) allows us to claim the visual aspect of the models changes only minimally. This leads us to the conclusion that models constructed are different, but equivalent from a practical point of view. Their intensity difference is practically null, and their shapes, although different, all have the same average distance to the other elements of the set. Hence, we believe one iterations is sufficient to build representative average models.

Concerning the invariance of the models to the reference images used in the method, Fig. 6 shows that the models built using different references seem to converge towards the same solution. Their shape difference presented in Fig. 6(a) of about $1.0 \mathrm{~mm}$ is low compared to the average distance of $2.5 \mathrm{~mm}$ between the models and the set elements, and just over the distance of $0.5 \mathrm{~mm}$ between successive average models. Figure 6(b) also presents a low disparity between the different models intensities.

\section{Conclusion}

We have presented a completely automatic method to build average anatomical models of the human brain using a set of MR images. To this end, brain shape variations between subjects were identified. Differences due to linear transformations were excluded, resulting in the quantification of pure morphological differences. The result is an average intensity and average shape image representative of the characteristics of the image set elements used for the construction. The coupling of such a high quality model with statistical information regarding normal deformations, such as the work of [17], could enrich the significance of statistical tests by adding intensity information, useful for example in detecting gliosis in T2 MR images, and would supply an important tool in the analysis of normal anatomy. 


\section{References}

1. Ruzena Bajcsy and S. Kovacic. Multiresolution elastic matching. Computer Vision, Graphics and Image Processing, 46(1):1-21, April 1989.

2. Fred L. Bookstein. Principal warps: Thin-plate splines and the decomposition of deformations. IEEE Transactions on Pattern Analysis and Machine Intelligence, 11(6):567-585, June 1989.

3. Fred L. Bookstein. Thin-plate splines and the atlas problem for biomedical images. In Information Processing in Medical Imaging, volume 511 of Lecture Notes in Computer Science, pages 326-342, Wye, United Kingdom, July 1991.

4. Fred L. Bookstein. Shape and the information in medical images: A decade of the morphometric synthesis. Computer Vision and Image Understanding, 66(2):97118, may 1997.

5. M.E. Brummer, R.M. Mersereau, R.L. Eisner, and R.R.J. Lewine. Automatic detection of brain contours in MRI data sets. IEEE Transactions in Medical Imaging, 12(2):153-166, June 1993.

6. Gary Christensen, Richard D. Rabbitt, and Michael I. Miller. 3D brain mapping using a deformable neuroanatomy. Physics in Medecine and Biology, 39:608-618, March 1994.

7. D. Louis Collins, Terry M. Peters, and Alan C. Evans. An automated 3D non-linear image deformation procedure for determination of gross morphometric variability in human brain. In Visualisation in Biomedical Computing, volume 2359 of SPIE Proceedings, pages 180-190, Rochester, October 1994.

8. Alan C. Evans, D. Louis Collins, S. R. Mills, E. D. Brown, R. LO. Kelly, and Terry M. Peters. 3D statistical neuroanatomical models from 305 MRI volumes. In IEEE Nuclear Science Symposium/Medical Imaging Conference, pages 18131817, 1993.

9. Alexandre Guimond, Gérard Subsol, and Jean-Philippe Thirion. Automatic MRI database exploration and applications. International Journal of Pattern Recognition and Artificial Intelligence, 11(8):1345-1365, December 1997.

10. Karl Heinz Höhne, Michael Bomans, Martin Riemer, Rainer Schubert, Ulf Tiede, and Werner Lierse. A volume-based anatomical atlas. IEEE Computer Graphics and Applications, pages 72-78, July 1992.

11. David G. Kendall. A survey of the statistical theory of shape. Statistical Science, $4(2): 87-120,1989$.

12. Eduard Pernkopf. Atlas d'anatomie humaine. Piccin, 1983.

13. Gérard Subsol, Jean-Philippe Thirion, and Nicholas Ayache. A general scheme for automatically building 3D morphometric anatomical atlases: applications to a skull atlas. In Medical Robotics and Computer Aided Surgery, pages 373-382, Baltimore, November 1995.

14. Jean Talairach and Pierre Tournoux. Co-Planar Stereotaxic Atlas of the Human Brain. Thieme Medical Publishers, New York, United-States, 1988.

15. Jean-Philippe Thirion. Fast non-rigid matching of 3D medical images. In Medical Robotics and Computer Aided Surgery, pages 47-54, Baltimore, November 1995.

16. P. Thompson and A. W. Toga. A surface-based technique for warping 3dimensional images of the brain. IEEE Transactions in Medical Imaging, 15:1-16, 1996.

17. Paul M. Thompson and Arthur W. Toga. Detection, visualization and animation of abnormal anatomic structure with a deformable probabilistic brain atlas based on random vector field transformations. Medical Image Analysis, 1(4):271-294, September 1997. 